\title{
Une collaboration internationale au plus haut niveau scientifique
}

Le cas de l'enseignement des sciences en Serbie

A top-level international scientific collaboration Science education in Serbia

Una colaboración internacional en el mayor nivel científico El caso de la

enseñanza de las ciencias en Serbia

\section{Stevan Jokic}

Traducteur : Robert Elbaz

\section{OpenEdition}

\section{Journals}

Édition électronique

URL : http://journals.openedition.org/ries/665

DOI : $10.4000 /$ ries.665

ISSN : 2261-4265

\section{Éditeur}

Centre international d'études pédagogiques

\section{Édition imprimée}

Date de publication : 1 septembre 2009

Pagination : 59-65

ISSN : 1254-4590

\section{Référence électronique}

Stevan Jokic, "Une collaboration internationale au plus haut niveau scientifique », Revue internationale d'éducation de Sèvres [En ligne], 51 | septembre 2009, mis en ligne le 01 septembre 2012, consulté le 01 mai 2019. URL : http://journals.openedition.org/ries/665;DOI : 10.4000/ries.665 


\title{
Une collaboration internationale au plus haut niveau scientifique
}

\author{
Le cas de l'enseignement des sciences en Serbie*
}

\author{
Stevan Jokić
}

\section{L'ÉTAT ACTUEL DE L'ENSEIGNEMENT DES SCIENCES}

La situation n'est guère satisfaisante en matière d'enseignement scientifique en Serbie, à tous les niveaux, parce que cet enseignement est essentiellement présenté comme un cours magistral qui comprend de très rares activités expérimentales effectuées par les élèves. Il faut ajouter que l'essentiel des ressources dont disposent professeurs et élèves sont les manuels scolaires, parfois des revues scientifiques telles que Young Physicist (Le Jeune physicien), la Chemical Review (Revue de chimie), Galaxy (Galaxie) et quelques ouvrages de vulgarisation scientifique. Internet est largement utilisé mais, selon ma propre estimation, seuls environ $10 \%$ des enseignants et des enseignants des disciplines scientifiques l'utilisent pour la préparation de leur cours ou pour des projets menés par leurs élèves. Les sites pédagogiques et scientifiques en serbe sont en effet très rares et les enseignants comme les élèves utilisent la plupart du temps des sites étrangers.

Nous disposons par ailleurs, dans presque chaque région, de centres scientifiques pour les élèves doués, dans lesquels est mise en œuvre une pédagogie par projet ou par problème. Les plus célèbres, financés par le gouvernement, sont les lycées mathématiques et le centre de la recherche scientifique Petnica. Toutefois, le nombre d'élèves de ces centres est bien trop faible si nous souhaitons mettre en place une société fondée sur la connaissance.

Cependant, un nombre de plus en plus élevé d'élèves, et pas seulement les plus doués, utilise cette approche par l'intermédiaire de matières optionnelles («Découverte pratique du monde», «Les gardiens de la nature», «Du jouet à l'ordinateur) et parfois de la méthode IBSE (Inquired-Based Science Education : enseignement scientifique fondé sur l'investigation) à l'école primaire.).

1. Article traduit par Robert Elbaz. 
Comme c'est le cas dans de nombreux autres pays, le nombre d'étudiants diplômés de l'enseignement supérieur scientifique ne cesse de diminuer. La raison en est probablement la caractéristique essentielle de la société actuelle, qui favorise un accès tout à la fois rapide et facile à des postes supérieurs, par l'intermédiaire d'activités dans les banques, la politique, le management, etc. L'une des preuves en est que l'on observe la création de nombreuses universités privées dans le domaine de la finance ou du management, de quelques unes dans le domaine de l'environnement et de l'informatique associée au management, mais aucune dans les domaines scientifique et technologique. D’un autre côté, les universités scientifiques et technologiques gouvernementales ne sont pas aussi bien équipées et ne pourraient propulser de façon satisfaisante davantage d'étudiants (et pas seulement un petit pourcentage des meilleurs) dans la compétition internationale pour la mise au point de nouvelles technologies.

\section{LES PROGRAMMES DE SCIENCES ACTUELS}

La convention de Bologne s'applique à l'université. Actuellement, les départements assurant la formation des enseignants proposent environ $60 \%$ de matières didactiques, méthodologiques, pédagogiques et psychologiques ou relatives à l'expérience pédagogique et $40 \%$ de matières d'enseignement général ou scolaire. Parmi celles-ci, la science occupe la seconde place avec environ $10 \%$. Cet enseignement est conduit par des professeurs de physique, de chimie et de biologie, essentiellement sous la forme de cours magistraux qui comportent parfois des démonstrations expérimentales mais fort peu d'expériences réalisées par les étudiants eux-mêmes. Les départements assurant la formation des professeurs de physique, de chimie et de biologie assurent environ $15 \%$ de matières didactiques, méthodologiques, pédagogiques et psychologiques ou relatives à l'expérience pédagogique et $85 \%$ de disciplines scientifiques. Là encore, la présentation sous forme de cours magistral domine mais les expériences menées de façon individuelle ou en groupe sont plus fréquentes, surtout en chimie et en biologie, moins en physique.

Les programmes scolaires contiennent, à l'école élémentaire, environ $15 \%$ de disciplines scientifiques, qui se présentent essentiellement sous la forme d'un cours magistral et de quelques démonstrations expérimentales. On utilise l'IBSE dans quelques cours optionnels tels que «Découverte du monde», "Les gardiens de la nature», etc. À l'école primaire, où les enfants sont âgés de 11 à 15 ans, l'enseignement scientifique concerne environ 20 à $25 \%$ du programme, alors qu'au lycée il en représente $35 \%$, sous la forme d'enseignements de chimie, de biologie, de physique et d'enseignement technique, essentiellement dispensés sous la forme de cours magistraux, rarement sous la forme de problèmes expérimentaux et de projets conduits par les élèves eux-mêmes. Dans 
les différents établissements du second degré technique, l'enseignement comporte $20 \%$ de chimie, de physique et de biologie au cours des deux premières années, ainsi que certaines matières technologiques spéciales, qui varient selon l'établissement, au cours des deux dernières années. Les expériences, les problèmes et les sujets n'y occupent pas encore suffisamment de place parce que nous n'avons pas changé de technologie au cours des vingt dernières années. Presque toutes les anciennes usines ont fermé et fort peu se sont créées dans le domaine des nouvelles technologies.

\section{QUE FAIRE POUR AMÉLIORER LA SITUATION?}

L'expression "société de la connaissance» est de plus en plus utilisée par le gouvernement, la communauté éducative et scientifique et la presse, tout comme elle est le sujet central de nombreux ateliers et conférences. Certaines stratégies ont été développées. Le gouvernement a promis d'équiper toutes les écoles serbes d'ordinateurs et de connexions au réseau. Le soutien financier des étudiants brillants, qui ont remporté les Olympiades mathématiques et scientifiques dans de nombreuses compétitions internationales, est également d'actualité. Ces mêmes étudiants sont également soutenus par la presse et la télévision. Cependant, tout cela n'est pas suffisant. Comme l'a déclaré Georges Charpak, prix Nobel de physique, à propos de la situation française : "Si un pays de soixante millions d'habitants ne recrute ses ingénieurs que dans des milieux qui représentent $10 \%$ de la population, c'est comme s'il se réduisait à un peuple de six millions d'habitants». La Serbie compte huit millions d'habitants, et la «société de la connaissance» ne pourra voir le jour que si la conception de l'enseignement scientifique repose sur les trois principes suivants, lancés aux États-Unis il y a plus de vingt ans : la science est la propriété de tous; les élèves étudient les sciences à l'école; moins signifie, parfois, plus. Ainsi, la stratégie mentionnée ci-dessus, qui ne touche que les élèves les plus doués, doit être complétée par une littéracie scientifique de tous les enfants.

C'est en ayant tout cela à l'esprit, ainsi que l'expérience américaine Hands-on ${ }^{2}$, celle de la France avec La main à la pâte, celle de la Suède avec NCF, celle de la Chine avec Learning by doing ${ }^{3}$, et d'autres, que nous avons essayé de changer cette situation en introduisant la méthode IBSE dans l'enseignement des sciences et en développant dès 2001 - de façon individuelle ou avec l'appui de la Société serbe de physique - la collaboration avec l'Académie des sciences française, grâce à l'aide inestimable des académiciens Pierre Léna et Yves Quéré et de l'équipe française de La main à la pâte.

2. Hands-on : comprendre en manipulant et en agissant (NdT).

3. Learning by doing: «Apprendre en faisant» (NdT). 
En tout premier lieu, notre stratégie a été d'atteindre la littéracie scientifique pour tous les enfants, en créant différentes ressources mises à la disposition des enseignants.

\section{LES OBJECTIFS ATTEINTS}

\section{La traduction d'ouvrages}

L'équipe française de La main à la pâte compte environ vingt personnes et dispose de l'appui de l'Académie des sciences française, du gouvernement français et de la communauté scientifique française. Cette aide de la communauté scientifique et des étudiants apportée aux enseignants prend la forme d'une présence au sein de la classe et d'une rédaction de ressources pédagogiques, c'est-à-dire, par l'intermédiaire d'une activité de suivi des enseignants, d'un véritable accompagnement. Cette idée était tout à fait inédite en Serbie et nous ne disposions pas, du moins au début, d'un soutien réellement ferme. Pour cette raison, nous avons décidé de traduire autant de livres créés à l'intention des enseignants et des parents par l'équipe de La main à la pâte que nous le pouvions. Ce faisant, en augmentant les ressources disponibles en langue serbe, nous avons rempli certaines conditions minimales. Grâce aux traductions, nous avons instauré une véritable communication entre les communautés éducatives scientifiques française et serbe. Grâce à de bonnes ressources éducatives et scientifiques, nous servons notre langue de la meilleure façon possible. Certes, elle est très peu diffusée mais elle a sa place dans la culture mondiale et nous contribuons ainsi à son existence ${ }^{4}$. Les livres Enseigner les sciences à l'école, Découvrir le monde à l'école maternelle aident, de la meilleure façon possible, les enseignants à mener à bien les activités pédagogiques dans leurs classes. Durant la traduction des livres de la série "Graines de Sciences», nous avons été aidés par un certain nombre d'universitaires, de chercheurs provenant de différents instituts et d'enseignants. Ainsi, d'une manière différente, nous avons mis en place une forme de suivi des enseignants.

\section{Un site serbe miroir du site web français La main à la pâte}

Huit années de travail nous ont permis de développer un vaste partenariat avec différentes instances : l'Institut Vinča de sciences nucléaires, la Société serbe de physique, le ministère de l'éducation serbe, le ministère des sciences et de la recherche serbe, Eurosciences, l'éditeur scolaire Zavod za udzbenike, la revue pédagogique hebdomadaire Prosvetni prelged, l'ambassade de France en Serbie, l'Université de Belgrade, l'Académie serbe des sciences et des arts, etc. Le professeur Nikola Hajdin, président de l'Académie serbe des 
sciences et des arts, au cours de la cérémonie de signature de l'accord sur les droits de traduction en serbe du site web La main à la pâte, a présenté ainsi cette collaboration : "Notre pays est le premier de la région à signer cette sorte d'accord avec l'Académie des sciences, membre de l'Institut de France. Ce groupe d'enthousiastes tente de promouvoir les projets de type Hands-On dans notre pays, projet que de nombreux pays développés ont incorporé à leur système éducatif en le transformant, de manière à former la jeunesse à la société fondée sur la connaissance [...] La version serbe du site Web contient environ 2000 pages. Nos enseignants peuvent utiliser un très grand nombre de modules correspondant à des activités de classe; trois projets pédagogiques ( «Sur les traces d'Ératosthène», «Le climat, la planète et moi », «l'Europe des découvertes»); des documents pédagogiques; un espace ouvert aux enseignants; un espace réservé aux questions posées aux scientifiques.

Ces modules sont très importants et constituent une forme d'aide à la réalisation de cours optionnels tels que «Découvrir le monde», qui a été mis en place à l'école primaire en 2003, ainsi que d'autres projets scientifiques.

Ces projets pédagogiques permettent une approche interdisciplinaire et la participation de nos enseignants et de nos élèves à différentes activités internationales. Il s'agit là d'un pas extrêmement important car, au-delà de l'aspect scientifique, ils auront l'occasion d'utiliser des langues étrangères et de créer une sorte de réseau, tout à la fois national et international.

\section{Les ateliers pour les enseignants et les autres activités}

$\mathrm{Au}$ cours des trois dernières années, nous avons mis en place des ateliers rassemblant environ trois mille enseignants de maternelle et du primaire sur l'ensemble de la Serbie. Il s'agissait de simulations d'activités pédagogiques. Les stagiaires ont travaillé sur les problèmes expérimentaux proposés par la méthode IBSE, rassemblant les enfants par groupes pouvant aller jusqu'à six élèves et disposant de matériaux qu'ils pouvaient facilement trouver autour d'eux, en mettant en place un protocole expérimental, des hypothèses, en conduisant des expériences, en élaborant des conclusions et en créant des affiches. Durant les Fêtes de la Science, plus de 1500 enfants, âgés de 4 à 11 ans, ont réalisé des expériences. En collaboration avec l'ambassade de France à Belgrade, l'Académie des sciences française et un centre scientifique de Grenoble, nous avons mis en place deux expositions à Belgrade, «La science à l'école : quelle histoire!» et "Nanotechnologies».

\section{L’activité internationale}

En tant que premier pays d'Europe du Sud-Est à avoir appliqué la méthode IBSE en matière d'enseignement des sciences, nous avons mis en place 
quatre écoles internationales couvrant cette zone géographique, ainsi que des conférences pour les ministres de l'Éducation des pays concernés.

Le projet serbe, inspiré de Hands-on (Ruka u testu), fait partie du consortium FIBONNACCI, mis en place à l'appel du groupe de recherche «La science et la société», dans le cadre du FP7 ${ }^{5}$.

Un accord de collaboration, dans les domaines de l'enseignement des sciences entre l'Académie des sciences française, l'Académie serbe des sciences et l'Université de Belgrade a été signé.

Un livre, Découvertes scientifiques dans la région des Balkans, est en préparation. Au cours des dernières décennies, les recherches conduites à l'échelle mondiale ont montré que l'histoire des sciences constitue un gigantesque réservoir où puiser pour améliorer le processus d'apprentissage des sciences, tout spécialement dans une perspective constructiviste, permettant de mettre en œuvre une approche fondée sur l'investigation scientifique dans les écoles primaires. $\mathrm{Au}$ cours de l'histoire, la région des Balkans s'est surtout fait connaître par les problèmes et les crises qui l'ont traversée. Nous aimerions montrer un autre aspect de notre région grâce à la présentation de contributions scientifiques issues des pays des Balkans, contributions qui seront utilisées pour impliquer les enfants dans un processus d'apprentissage fondé sur l'investigation scientifique.

\section{Perspectives}

\section{DANS UN PROCHE AVENIR}

Malgré la création des ressources mentionnées plus haut et celle d'ateliers avec les enseignants, nous avons vu que des kits pédagogiques doivent absolument être créés et offerts à chaque établissement et à chaque enseignant. C'est pour cette raison que, au cours des deux prochaines années, nous fournirons un certain nombre de ressources spécifiques pour la salle de classe, comprenant des kits pédagogiques et des instructions pour les enseignants. Dans cent écoles primaires couvrant l'ensemble du territoire serbe, nous espérons créer des salles expérimentales qui donneront aux enseignants un modèle de recherche et de mise en œuvre du processus d'apprentissage fondé sur l'investigation, dans un local adapté doté des outils appropriés. Ces kits pédagogiques, spécialement conçus pour cette approche, couvriront certains sujets des programmes de sciences et de technologie.

La collaboration avec la Faculté de formation des enseignants de Belgrade est d'ores et déjà acquise, et de jeunes assistants-chercheurs feront partie de notre équipe. Nous essaierons de créer des thèses de doctorat sur ce sujet et d'introduire davantage de modules de type IBSE dans les programmes scientifiques.

5. FP7 : Seventh Framework Programme - septième programme-cadre mis en œuvre par la Commission Européenne $(\mathrm{NdT})$. 
La méthode IBSE est appliquée dans environ $10 \%$ des écoles primaires serbes et notre projet est internationalement reconnu. Nous espérons qu'elle sera officiellement introduite par le ministère de l'éducation dans toutes les écoles serbes. Le rôle d'Internet et des technologies de l'information à orientation pédagogique peut être extrêmement utile par leur capacité à disséminer les idées relatives à cette méthode d'enseignement des sciences dans les écoles primaires.

\section{BiBLIOGRAPHIE}

Mathematical High School, Belgrade : http://www.mg.edu.rs/engleski/index.html/. Petnica Science Centre : http//pi.petnica.rs/.

Robert Germinet, L'Ingénieur au chevet de la démocratie, préface de Georges Charpak, Odile Jacob, 2004 (édité et traduit en serbe par Stevan Jokic).

Stevan Jokić a traduit, dirigé ou co-dirigé les livres suivants :

Georges Charpak, La main à la pâte, Flammarion, Paris 1996, (Društvo fizičara Srbije, Beograd, 2001 i 2003);

Catala I. (dir.), Léna P. (dir.), Quéré Y. (dir.), Graines de sciences 1, Le Pommier, 1999 (Zrnca nauka 1, Društvo fizičara Srbije, Beograd, 2003);

Catala-Blanc I. (dir.), Jasmin D. (dir.), Léna P. (dir.), Graines de sciences 2, Le Pommier, 2000 (Zrnca nauka 2, Zavod za udžbenike i nastavna sredstva-Društvo fizičara Srbije, Beograd, 2004);

Jasmin D. (dir.), Boucharad J. -M. (dir.), Léna P. (dir.), Graines de sciences 3, Le Pommier, 2001 (Zrnca nauka 3, Zavod za udžbenike i nastavna sredstva, Beograd, 2004);

CNDP, Enseigner les sciences à l'école, Paris, 2002 (Predavanje nauka u školi, Zavod za udžbenike i nastavna sredstva, Beograd, 2004);

Wigenbus D. (dir.), Bouchard J. -M. (dir.), Léna P. (dir.), Graines de sciences 4, Le Pommier, 2001 (Zrnca nauka 4, Zavod za udžbenike i nastavna sredstva, Beograd, 2004);

Wigenbus D. (dir.), Salviat B. (dir.), Julia M. (dir.), Graines de sciences 5, Le Pommier, 2003 (Zrnca nauka 5, Zavod za udžbenike i nastavna sredstva, Beograd, 2008);

Jasmin. (dir.), L'Europe des découvertes, Le Pommier, 2004 (Evropa otkrića, Zavod za udžbenike i nastavna sredstva, Beograd, 2007);

Découvrir le monde à l'école maternelle, collectif, CNDP 2002 (Otkrivanje sveta predškolskoj ustanovi, Prosvetni pregled, Beograd, 2007).

Site web serbe La main à la pâte (Ruka u testu) : http://rukautestu.vinca.rs/.

South-East European Summer School for Hands On Primary Science Education, 1-4, ed. Stevan Jokić, Vinca Institute 2006-2009, voir : http://rukautestu.vinca.rs/handson4/.

Stevan Jokić, "Les Sciences à l'école primaire : «La main à la pâte», un projet pour l'Europe du Sud-est ?», contribution à la $3^{\mathrm{e}}$ conférence informelle des ministres de l'Éducation des pays du Sud-Est européen, "Strengthening the teaching profession as a driving force for development" (Renforcer la profession d'enseignant pour en faire le moteur du développement), Istanbul - Turquie, 24-25 avril 2004. 
